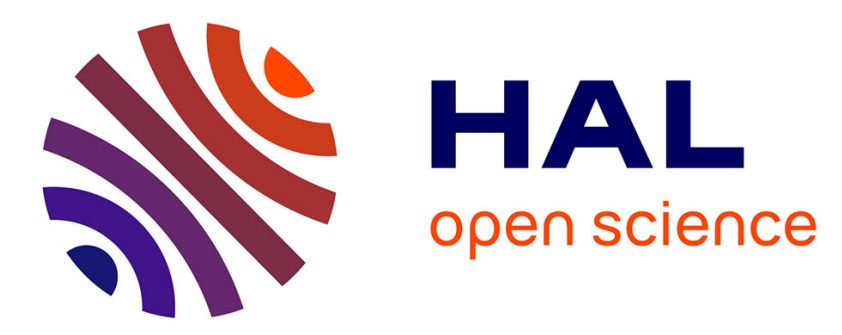

\title{
Artificial aggregation of domestic appliance currents for NILM evaluation
}

\author{
Mohamad Nour, Yves Raingeaud, Philippe Ravier, Guy Lamarque, \\ Jean-Charles Le Bunetel
}

\section{- To cite this version:}

Mohamad Nour, Yves Raingeaud, Philippe Ravier, Guy Lamarque, Jean-Charles Le Bunetel. Artificial aggregation of domestic appliance currents for NILM evaluation. EEEIC INTERNATIONAL CONFERENCE ON ENVIRONMENT AND ELECTRICAL ENGINEERING, Sep 2021, BARI, Italy. hal-03513298

\section{HAL Id: hal-03513298 \\ https://hal.science/hal-03513298}

Submitted on 5 Jan 2022

HAL is a multi-disciplinary open access archive for the deposit and dissemination of scientific research documents, whether they are published or not. The documents may come from teaching and research institutions in France or abroad, or from public or private research centers.
L'archive ouverte pluridisciplinaire HAL, est destinée au dépôt et à la diffusion de documents scientifiques de niveau recherche, publiés ou non, émanant des établissements d'enseignement et de recherche français ou étrangers, des laboratoires publics ou privés. 


\section{Artificial aggregation of domestic appliance currents for NILM evaluation}

\author{
Mohamad Nour \\ GREMAN \\ Université de Tours \\ Tours, France \\ mohamad.nour@univ-tours.fr \\ Yves Raingeaud \\ GREMAN \\ Université de Tours \\ Tours, France \\ yves.raingeaud@univ-tours.fr
}

\author{
Philippe Ravier \\ PRISME \\ Université d'Orléans \\ Orléans, France \\ philippe.ravier@univ-orleans.fr \\ Guy Lamarque \\ PRISME \\ Université d'Orleans \\ line 4: Orleans, France \\ guy.lamarque@univ-orleans.fr
}

\author{
Le Bunetel Jean-Charles \\ GREMAN \\ Université de Tours \\ Tours, France \\ jean-charles.lebunetel@univ-tours.fr
}

\begin{abstract}
Dedicated Nonintrusive Load Monitoring (NILM) datasets are needed to test any new NILM process. This, however, introduces limitation, since datasets can be lacking in terms of load diversity and multi-load signals. The first problem can be solved by acquiring data from other datasets. The second one requires real measurements of said signals. With the intention of solving the second limitation, we present a new method to create artificial multi-load signals using real individual load signals. The method is able to place the signals at any position relative to one another. Its performance is tested by comparing the resulting artificial signal with the real signal. The results shows strong similarities between the real and artificial signals. An application in ON-OFF transition counts is introduced showing the usefulness of the proposed artificial aggregation approach for any deep evaluation of event detectors in NILM.
\end{abstract}

Keywords - NILM, multiple load detection, current signal mixing, dataset expansion, artificial aggregation

\section{INTRODUCTION}

Facing the ever-growing energy demand worldwide, countries are moving towards sustainable development, which translate to finding more efficient and conservative ways to consume energy. Buildings of different kinds (residential, commercial and public service) are a very big target for this new development, since they consumed around $49 \%$ of the world's end-use electricity (2017) [1]. Additionally, the building's energy consumption and greenhouse gas emissions may have doubled or tripled during the past decade [2].

In the direction of this goal, the installation of smart grids and consequently that of the smart meters has seen a rise recently [3]. The smart meter is a counter installed at the level of the main breaker in the building or household that records the overall consumption, active and reactive power and sends this information to the central system and the consumer. This has benefits for the provider and the client. The provider accesses the real consumption and can charge the clients based on it. In addition, the provider can send different offers to the clients that best suits their consumption behaviors. The client benefits by having his awareness raised about his consumption and which is a drive to improve his energy management at the consumer's side. However, [4] shows that pure consumption values do not automatically lead to better management. Instead, these data need to be transformed into practical easy to understand information that the consumer can exploit.

Next comes the idea of adding an extra step to the smart meter: smart energy management. This will transfer to the client the detailed state and consumption of the different appliances individually in addition to different suggestions to optimize the usage of these appliances. For example, the smart management suggests to the client to delay the usage of the washing machine by one hour in order to start it at the off-peak hours instead of starting it at the peak-hours. To achieve this management, a hidden step must be performed: identification of the different active loads. Several approaches can be used. Two well-known approaches are: (a) installing a current sensor on each appliance or group of appliances [5] (b) installing one current sensor at the main breaker. The names intrusive and nonintrusive load monitoring (ILM and NILM) are associated to the two approaches respectively.

The field of NILM was first introduced in 1980 by Hart [6]. Despite being three decades old, the interest in it had recently emerged. The process measures and analyzes the signal at the main breaker level (aggregated signal), in the interest of delivering a feedback to the consumer about the state of the different active and inactive loads.

The NILM process generally follows three main steps. The first step is the event detection, which refers to the task of locating transitions (ON-OFF appliances, change in the operation mode ...) in the aggregated signal. Kernel clustering [7], hidden Markov model [8], and goodness of fit are some examples of said methods. The second step extract specific signatures, also known as features, from the signal. Said features can be extracted from the transient phases, permanent phases, or both phases [9]. Finally, the last step separate the aggregated wave into individual load signals that describe the type and behavior of the corresponding appliance. A review of the different disaggregation algorithm can be found in [10] [11] [12] [13].

Two different algorithms can be seen in a general NILM process: the event detection and the identification algorithm. A large data pool is required to test the performance of these two. For that purpose, several datasets of aggregated and disaggregated load signals have been developed, each with different appliance variety, sampling frequency, signal duration...REDD [14], BLUED [15], PLAID [16], WHITED [17] and COOLL [18] are all examples of the available public NILM datasets. Although a large number of database exists, some with large appliance varieties, they mostly host individual load waveforms and in the case of multi-load signals, they focus on long duration measurements with random activation time instants of each appliance and often without having ON/OFF instants ground truth. As a result, two issues are present: 1) the shortage of multi-appliance waveforms 2) the inability to control the startup of one appliance relative to the others. 
It is possible to redeem this shortage of multi-appliance signals by performing manual measurements of these signals. However, the number of possible mixed signals increases with the number of loads: this includes the possible number of loads in one signal plus the number of possible usage scenarios. For these reasons, it is interesting to develop a program that "artificially" creates any possible scenario from a set of individual signals. The program should be able to create any desired scenario from the given individual load signals.

We present in this paper a solution to this problem that allows combining a set of individual appliance current into one longer multi-appliance current signal. Furthermore, the method allows placing each appliance at any position relative to the others. The algorithm is fast, simple, and the artificially mixed signal strongly resembles the real mixed signal. In this paper, the word aggregate, mix and combine are interchangeable.

The paper is divided into 5 sections: section 2 presents the algorithm with the different preparation needed to correctly use it. Section 3 compare and discusses the artificial results and the natural ones. The next section describes some application results of the algorithm. The last section draws the conclusion as well as opens the door to new perspectives.

\section{ARticial AgGREGATION}

\section{A. Aggregation algorithm}

The algorithm requires a minimum of two signals to do the mixing. It starts by assuming that one signal is fixed and the other signals will be added to it. Let $I_{0}$ be the fixed signal and $I_{i}$ be any of the other signals. The proposed algorithm is able to add the signals at any position in $I_{0}$.

For the sake of simplicity, we will assume the case of mixing two signals. Let $V_{0}, I_{0}, V_{1}$ and $I_{1}$ be the fixed signal voltage and current, and the second signal voltage and current respectively. The algorithm is as follow:

1. Voltage synchronization: using a simple zero crossing detection program, we detect the descending zeros in $V_{0}$ and $V_{1}$. Let $Z_{0}$ and $Z_{1}$ be the location of these zeros.

2. Current offset compensation: calculate the offsets of the two current signals $I_{0}$ and $I_{1}$. Let $m_{0}$ and $m_{1}$ be these two offsets. Then subtract each offset from its corresponding signal.

3. Pick any position from $Z_{0}$ (position number 9 for instance) and pick the first position in $Z_{1}$. Then add the two signals in matrix form as follow (we assume the signals to be column vectors):

$$
I_{\text {total }}=\left[\begin{array}{c}
I_{0}\left(1: Z_{0}(i)\right) \\
I_{0}\left(Z_{0}(i): \text { end }\right)+I_{1}\left(Z_{1}(1): \text { length }\left(I_{0}\right)\right) \\
I_{1}\left(\text { length }\left(I_{0}\right): \text { end }\right)
\end{array}\right]
$$

\section{B. Voltage synchronization}

The first step in the algorithm consists of locating similar positions in the voltages of the different signals. Then we ensure that the positions of the fixed signal, on which we will add the other signals, and those of the other signals are of similar nature (in the algorithm, the nature of these positions is "descending zeros". However, they can be ascending zeros, maxima, minima ...).

This synchronization step is required to obtain an acceptable mixed signal. Otherwise, we risk of causing signal interference and consequently cause the signals to subtract from one another instead of adding up.

Consider the two current signal in Fig. 1 .We shift the two signals in a way that the two corresponding voltage do not start at similar positions Fig. 2a. If we add the two currents we obtain the signals in Fig. 2c. We observe a very clear interference phenomenon. On the other hand, if we shift the two signals so that the two voltages start at similar positions (a maximum in this case) Fig. 2b, then the signals add up instead of interfering with one another Fig. 2d.

\section{Offset Compensation}

The miss calibration of the different measurement equipment introduce an offset in the measured current. If we follow (1), we see that $I_{\text {total }}$ is made from three parts: the first part comes from $I_{0}$, the second part is the sum of a portion of $I_{1}$ and $I_{0}$ and the third part comes from $I_{1}$. This implies that the offset of $I_{\text {total }}$ is inconsistent throughout the signal; it starts as the offset of $I_{0}$ then the offset of $I_{1}$ is added and finally we return to that of $I_{1}$.

We consider the two current signals in Fig. 3. The offset of the two signals is $0.15 \mathrm{~A}$. We will add $I_{1}$ to $I_{0}$ around the instant $t=3 \mathrm{~s}$. The signal $I_{0}$ ends at $t=6 \mathrm{~s}$ and the signal $I_{1}$ remains until $t=9 s$.

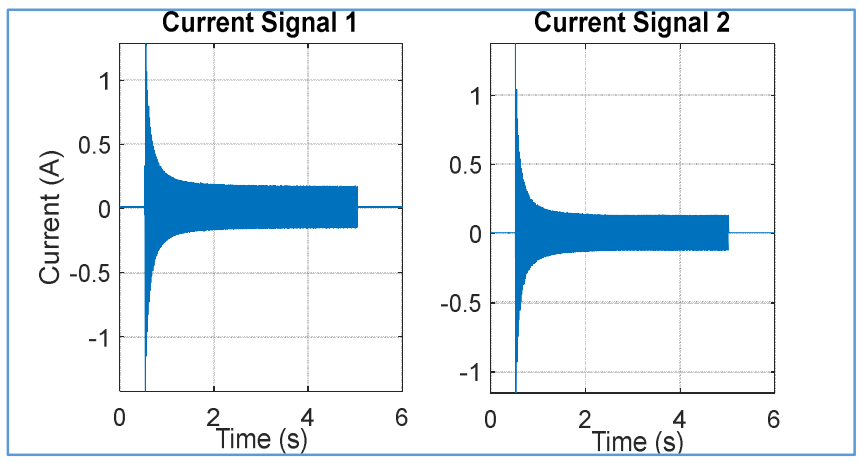

Fig. 1. Waveform of the two current signals

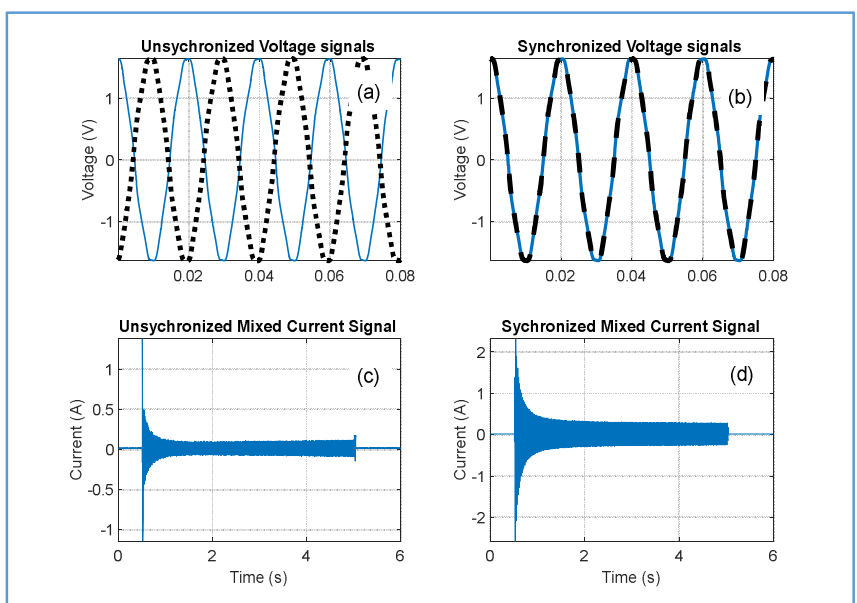

Fig. 2. (a) Unsynchronized Voltage signals (b) Synchronized voltage signals (c) Resultant current with unsynchronized voltage (d) Resultant current with synchronized voltage. 
Without removing the offsets from the signals we have two sudden transitions, the first around $t=3 \mathrm{~s}$ and the second at $t=6 \mathrm{~s}$ which corresponds to the instant of adding $I_{1}$ and the instant of ending $I_{0}$ respectively Fig. $4 \mathrm{a}$ and $4 \mathrm{c}$. Such sudden jump could lead to errors in the event detection phase of the NILM. This is particularly apparent when we mix signals of small power consumption. On the other hand, the total current after removing the offsets (Fig. 4b and 4d) does not exhibit any of these sudden transitions.

\section{REAL AND ARTIFICIAL SIGNAL COMPARISON}

\section{A. Setup}

Using the setup in [19], we measure the voltage and current of two appliances individually (Fig. 5) and when they are active at the same time. The two appliance are a drill and a jigsaw. Each signal is 7 seconds length; the active part of the signal starts at around $0.5 \mathrm{~s}$ and ends around $6.5 \mathrm{~s}$. The signals are sampled at $10 \mathrm{kHz}$ and they all scaled with the same scale factor. Using the two individual signals, we apply the algorithm to construct the artificial aggregated signal.

\section{B. Comparison procedure}

\section{1) Visual comparison}

We plot the real and artificial aggregated signals in the following time intervals $[0.5 \mathrm{~s} ; 0.55 \mathrm{~s}]$ and $[3.5 \mathrm{~s} ; 3.55 \mathrm{~s}]$ (Fig. $7)$. The choice of these two intervals is to represent the form of the two currents in the transient and permanent phase respectively. A quick observation of the form of the two signals shows a strong resemblance between the two signals.

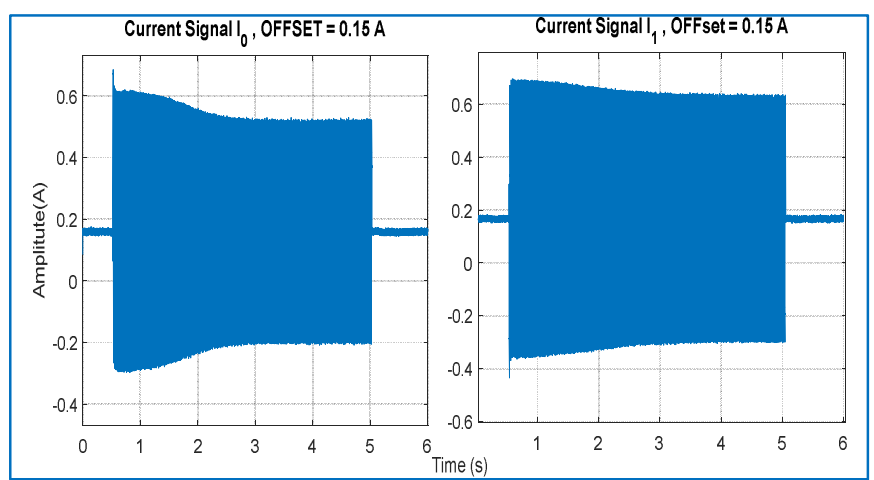

Fig. 3 Waveform of the two current signals with offset

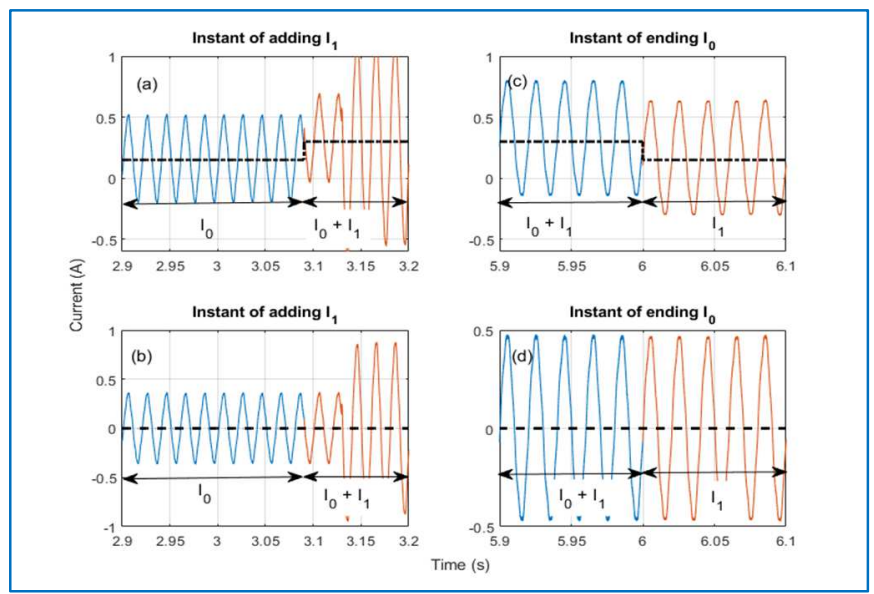

Fig. 4 (a) Instant of adding $I_{1}$ without offset compensation (b) Instant of adding $I_{1}$ with offset compensation (c) Instant of ending $I_{0}$ without offset compensation (d) Instant of ending $I_{0}$ with offset compensation

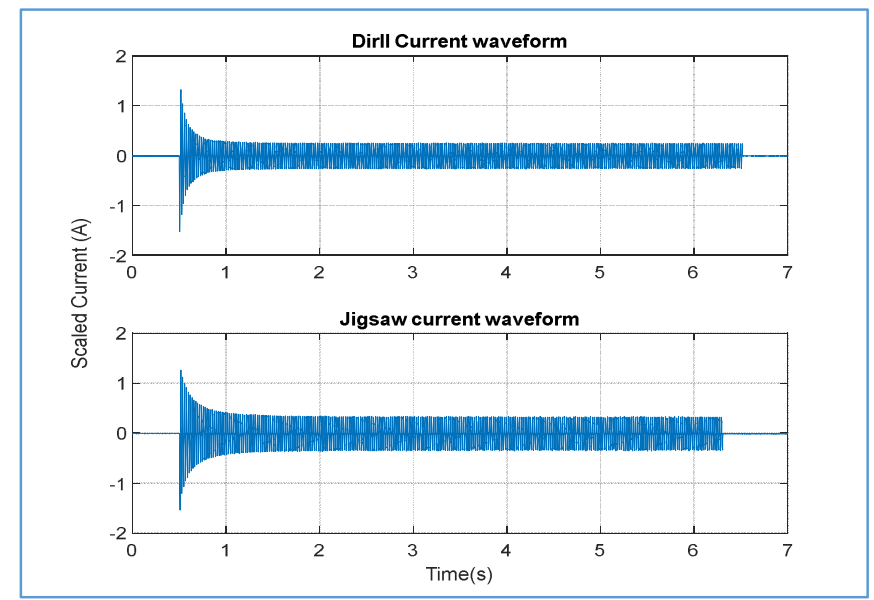

Fig. 5. Drill and Jigsaw individual current waveform

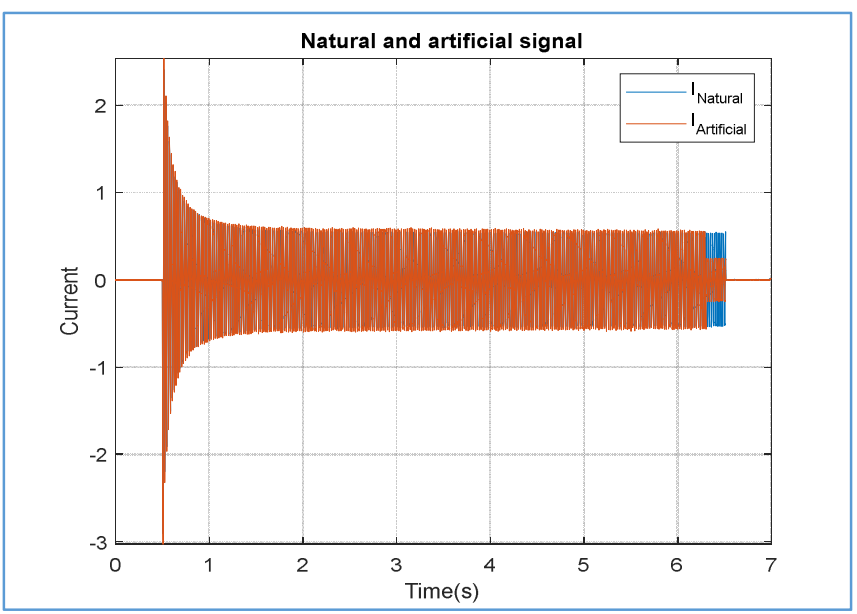

Fig. 6 Current waveform of the real and artificial aggregation

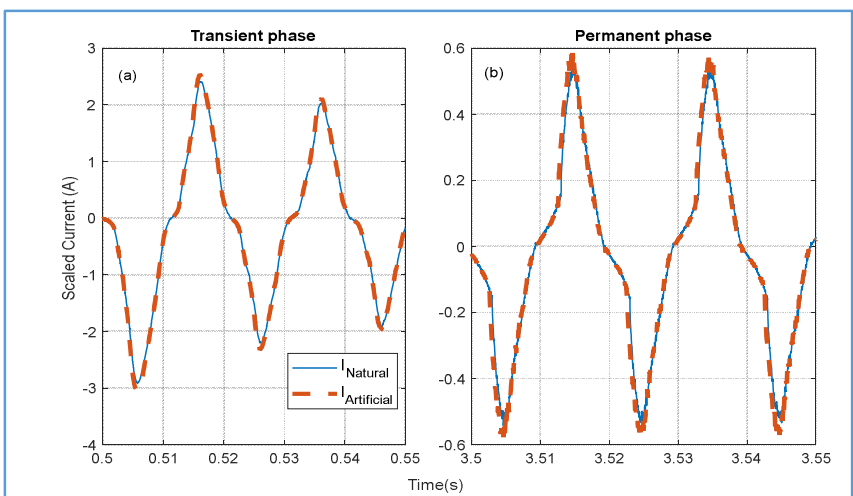

Fig. 7 (a) Start of the transient phase of the real and artificial mixed signals (b) the permanent phase of the real and artificial signal in the time interval $[3.5 \mathrm{~s}, 3.55 \mathrm{~s}]$

\section{2) Numerical comparison}

For the real and artificial signal comparison, we compare the transient phases and permanent phases separately and then we compare the whole signals. We use two tests. In the first test, we calculate the Root Mean Square (RMS) of each signal and compute the percentage of the real and artificial RMS difference relative to the real RMS.

The second test goes by computing the Fourier coefficients for each period in each signal, then comparing the group of coefficients of the real signals with those of the artificial one. For the second test, we apply the discrete fast Fourier transform on each period of the signal. We choose to consider 
the first five harmonics with the DC component. The fundamental frequency of the signals is $50 \mathrm{~Hz}$, the sampling frequency is $10 \mathrm{kHz}$ and each signal has $7 \mathrm{~s}$ duration. As a result, we obtain around 350 periods and, consequently, around 350 coefficients for each harmonic. We choose to calculate the Root Mean Square Error (RMSE) between the real and artificial coefficient, and then compute the percentage of the RMSE relative to the mean value of the harmonic of the real signal.

The results of the first test are in TABLE I and the results of the second test are in TABLE II. The metrics $R, M$ and $P$ refers to the RMSE expressed in A, the mean value of the harmonic in the real signal and the percentage respectively.

From the results of the first test (TABLE I), we find that the RMS of the artificial signal is around that of the real signal with a variation margin of around $5 \%$. The margin is different if we consider different part of the signal. However, the margin of the entire signal is around $4-5 \%$.

In the second test, we computed, for each harmonic, the RMSE between the real and artificial harmonic. The RMSE describes the mean value of the deviation between the two, thus comparing this mean to the average of the real harmonic is capable of giving an estimate of how different the artificial harmonics form the real ones. In TABLE II we note that the average of even Fourier coefficients is very small, almost near zero, average value, relative to the odd harmonics that hosts the majority of the signal's power, which, explains the big percentage for each of these frequencies for every part of the signal. On the other hand, the percentage of the odd frequencies appears to fluctuate around $5-8 \%$ throughout the different parts of the signal, except for the $150 \mathrm{~Hz}$ harmonics in the permanent part and whole signal that fluctuate around $12 \%$ and $10 \%$ respectively

TABLE I. THE COMPARISON RESULTS OF THE FIRST TEST

\begin{tabular}{|l|c|c|c|}
\hline Signal Part & Transient & Permanent & Whole Signal \\
\hline RMS (artificial signal) & 0.4669 & 0.2931 & 0.3126 \\
\hline RMS (real signal) & 0.4527 & 0.2742 & 0.2996 \\
\hline Percentage & $3 \%$ & $7 \%$ & $4 \%$ \\
\hline
\end{tabular}

TABLE II. THE RESULTS OF THE SECOND COMPARISON TEST

\begin{tabular}{|c|c|c|c|c|}
\hline \multirow{3}{*}{ Harmonic } & \multirow{2}{*}{ Metric } & \multicolumn{3}{|c|}{ Signal Part } \\
\cline { 2 - 5 } & & $\begin{array}{c}\text { Transient } \\
\text { Phase }\end{array}$ & $\begin{array}{c}\text { Permanent } \\
\text { Phase }\end{array}$ & $\begin{array}{c}\text { Whole } \\
\text { Signal }\end{array}$ \\
\hline \multirow{3}{*}{$0 \mathrm{~Hz}$} & $\mathrm{R}$ & 0.0035 & 0.0014 & 0.0019 \\
\cline { 2 - 5 } & $\mathrm{M}$ & 0.0069 & 0.0007 & 0.0019 \\
\cline { 2 - 5 } & $\mathrm{P}$ & $50 \%$ & $172 \%$ & $101 \%$ \\
\hline \multirow{3}{*}{$50 \mathrm{~Hz}$} & $\mathrm{R}$ & 0.0219 & 0.024 & 0.0232 \\
\cline { 2 - 5 } & $\mathrm{M}$ & 0.5704 & 0.3362 & 0.4053 \\
\cline { 2 - 5 } & $\mathrm{P}$ & $4 \%$ & $6 \%$ & $5 \%$ \\
\hline \multirow{3}{*}{$50 \mathrm{~Hz}$} & $\mathrm{R}$ & 0.0032 & 0.0012 & 0.0017 \\
\cline { 2 - 5 } & $\mathrm{M}$ & 0.007 & 0.0015 & 0.0025 \\
\cline { 2 - 5 } & $\mathrm{P}$ & $45 \%$ & $80 \%$ & $70 \%$ \\
\cline { 2 - 5 } & $\mathrm{R}$ & 0.0132 & 0.0141 & 0.0138 \\
\cline { 2 - 5 } & $\mathrm{M}$ & 0.1874 & 0.1185 & 0.1318 \\
\hline \multirow{3}{*}{$200 \mathrm{~Hz}$} & $\mathrm{P}$ & $7 \%$ & $12 \%$ & $10 \%$ \\
\cline { 2 - 5 } & $\mathrm{R}$ & 0.0017 & 0.0012 & 0.0013 \\
\cline { 2 - 5 } & $\mathrm{M}$ & 0.0031 & 0.0013 & 0.0017 \\
\hline \multirow{3}{*}{$250 \mathrm{~Hz}$} & $\mathrm{P}$ & $54 \%$ & $90 \%$ & $79 \%$ \\
\cline { 2 - 5 } & $\mathrm{R}$ & 0.0044 & 0.0021 & 0.0026 \\
\cline { 2 - 5 } & $\mathrm{P}$ & 0.0506 & 0.0405 & 0.0425 \\
\hline \multirow{3}{*}{} & & $8 \%$ & $5 \%$ & $6 \%$ \\
\hline
\end{tabular}

\section{APPLICATION}

The goal behind the algorithm is to first, extend the available datasets to fill the shortage of multi-appliance signals that exists in the current available NILM dataset.

Second is to have control over the starting instants of the different loads relative to one another. A good usage of this control is the placement of the startup and/or shutdown instants of two or multiple loads in close proximity. This in return will enable us to test the effectiveness of the NILM algorithm in critical scenarios like when two loads start up and/or shut down in close time intervals.

Lastly, the algorithm enable the user to mix appliance of different power consumption. With this type of mixing, it is possible to test the NILM when one load of high power consumption is operating with another load with significantly lower power consumption. The purpose is to note whether the NILM algorithm will detect two or one load.

To illustrate the second and third goal of the algorithm we consider the following example by taking three loads from the COOLL dataset: a $350 \mathrm{~W}$ jigsaw, a $500 \mathrm{~W}$ drill and a $15 \mathrm{~W}$ compact fluorescent light. First we combine the drill current with that of the jigsaw ten times, we start by placing the startup of jigsaw 10 periods in advance to that of the drill, then we repeat the process with 9 periods and so on until the startup of the two loads is at the same instant. Fig. 8 shows the first and last signal in the set.

If we apply an event detection algorithm, then it should detect four events for each signal in the set: two startups and two shutdowns. We choose to apply the HAND algorithm described in [20]. We choose a threshold 0.5. The detection results are in TABLE III.

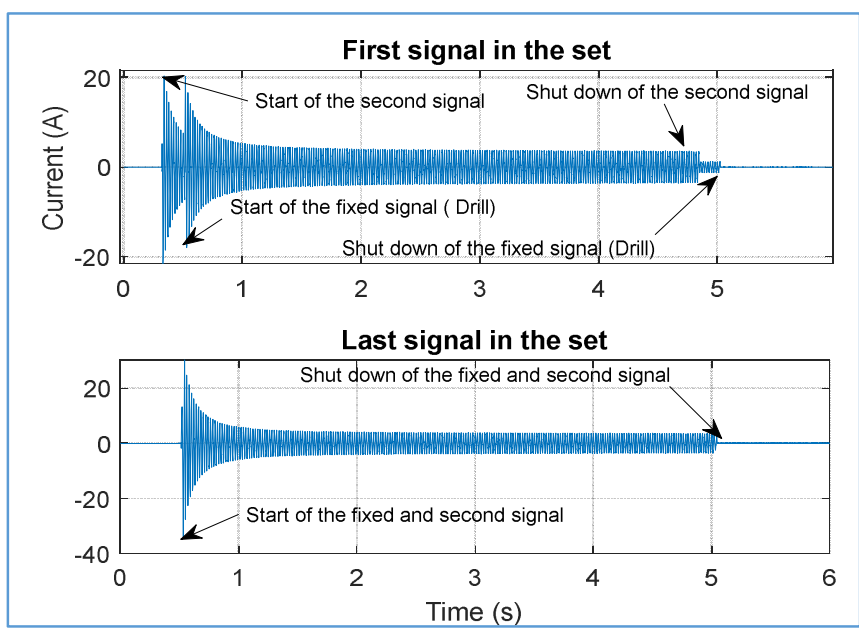

Fig. 8 The Current waveform of the first and last signal in the set of Drill and jigsaw combination

TABLE III. COUNTS OF THE TRANSITIONS IN THE DIFFERENT SIGNALS IN THE SET OF DRILL AND JIGSAW COMBINATION

\begin{tabular}{|c|c|c|c|c|c|c|c|c|c|c|}
\hline \multirow{2}{*}{ Transition } & \multicolumn{7}{|c|}{ Signal Number } \\
\cline { 2 - 13 } & $\mathbf{1}$ & $\mathbf{2}$ & $\mathbf{3}$ & $\mathbf{4}$ & $\mathbf{5}$ & $\mathbf{6}$ & $\mathbf{7}$ & $\mathbf{8}$ & $\mathbf{9}$ & $\mathbf{1 0}$ \\
\hline Startup & 1 & 1 & 1 & 1 & 1 & 1 & 1 & 1 & 1 & 1 \\
\hline Shutdown & 2 & 2 & 2 & 2 & 2 & 2 & 1 & 1 & 1 & 1 \\
\hline Total & 3 & 3 & 3 & 3 & 3 & 3 & 2 & 2 & 2 & 2 \\
\hline
\end{tabular}


The results shows that the HAND algorithm with the 0.5 threshold did not succeed in detecting the correct number of events for each signal. This has multiple implications: 1) the parameters of the event detection algorithm needs changing when the startups and/or shutdowns are in close proximity to one another 2) There is a certain minimum distance between two consecutive events that we cannot go below, otherwise the algorithm will consider the two events as one. This distance changes with the algorithm and its parameters.

Second, we combine the lamp signal with the drill signal. We place the startup of the lamp signal at 20 periods in advance to the shutdown of the drill and then repeat the same steps as before. Fig. 9 shows the first and last signal in the set. We apply the HAND algorithm with threshold 0.1 for achieving sufficient sensitivity required by the low power of the lamp. The result of the event detection are in TABLE IV.

The HAND detected the shutdown transitions correctly. However, the startup transitions are all down by one. This implies that the change caused by the startup of the lamp did not have a strong impact. It was masked by the much stronger permanent phase of the drill. In other word, if two appliances are active, the ratio of power consumption between the two should not go below a certain minimum, otherwise the algorithm will fail to detect the appliance, which consumes the least power.

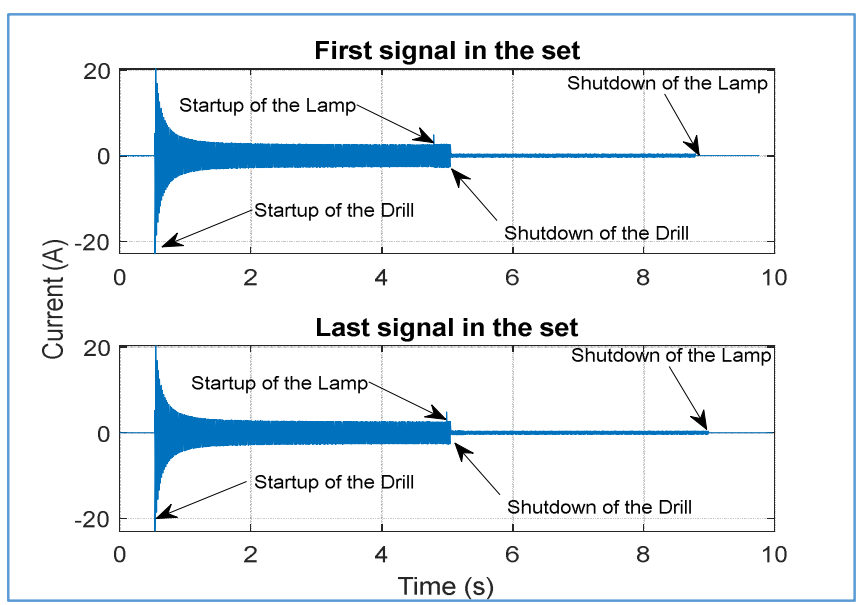

Fig. 10 Current waveform of the first and last signal in the set of lamp and drill combination

TABLE V. COUNT OF THE TRANSITIONS IN THE DIFFERENT SIGNALS IN THE SET OF LAMP AND DRILL COMBINATION

\begin{tabular}{|c|c|c|c|c|c|c|c|c|c|c|}
\hline \multirow{2}{*}{ Transition } & \multicolumn{10}{|c|}{ Signal Number } \\
\cline { 2 - 12 } & $\mathbf{1}$ & $\mathbf{2}$ & $\mathbf{3}$ & $\mathbf{4}$ & $\mathbf{5}$ & $\mathbf{6}$ & $\mathbf{7}$ & $\mathbf{8}$ & $\mathbf{9}$ & $\mathbf{1 0}$ \\
\hline Startup & 1 & 1 & 1 & 1 & 1 & 1 & 1 & 1 & 1 & 1 \\
\hline Shutdown & 2 & 2 & 2 & 2 & 2 & 2 & 2 & 2 & 2 & 1 \\
\hline Total & 3 & 3 & 3 & 3 & 3 & 3 & 3 & 3 & 3 & 2 \\
\hline
\end{tabular}

\section{CONCLUSION}

In this paper, we describe a new algorithm to combine individual current signals into one multi-current signal. The algorithm requires the voltage signals as well as the current signals, and it requires the calculation of the offsets. The algorithm is fast and simple, and the results show similarities to the real combined signals.

In the future, we would tune the thresholds and improve detection algorithm for better transition count results and expand the algorithm to add more than two signals. In addition, to test how the algorithm fairs when we combine long signals.

\section{REFERENCE}

[1] IEA - Statistics (2014) World: electricity and heat for 2014. Available online at: https://www.iea.org/statistics/statisticssearch (accessed on 7 July 2017).

[2] IPCC (2014) Climate Change 2014: Mitigation of Climate Change. Contribution of Working Group III to the Fifth Assessment Report of the Intergovernmental Panel on Climate Change, Cambridge University Press, Cambridge, United Kingdom and New York, NY, USA.

[3] Touzene, A., Yahyai, S.A. and Oukil, A. (2019) 'Smart grid resources optimisation using service oriented middleware', International Journal of Computer Applications in Technology, Vol. 59, No. 1, pp.53-63.

[4] Kelly, J. and Knottenbelt, W. (2016) 'Does disaggregated electricity feedback reduce domestic electricity consumption? A systematic review of the literature', Proceedings of the 3rd International Conference NILM Workshop, Vancouver, Canada.

[5] Burbano Acuña, M. D. (2015). Intrusive and non-intrusive load monitoring (a survey). Latin-American Journal of Computing, Systems Engineering, National Polytechnic School, Ecuador, 2(1). (Cité en page 18).

[6] George William Hart, "Nonintrusive appliance load monitoring," Proceedings of the IEEE, vol. 80, no. 12, pp. 1870-1891, 1992.

[7] M. Volpi, D. Tuia, G. Camps-Valls and M. Kanevski, "Unsupervised change detection by kernel clustering", Proc. SPIE, vol. 7830, 2010.

[8] V. Perduca, G. Nuel et al., "Hidden Markov Model Applications in Change-Point Analysis", arXiv Journal, preprint arXiv: 1212.1778, 2012

[9] A. Faustine, N. H. Mvungi, S. Kaijage, and K. Michael, "A survey on non-intrusive load monitoring methodies and techniques for energy disaggregation problem," CoRR, vol. abs/1703.00785, 2017. [Online].Available: http://arxiv.org/abs/1703.00785.

[10] Hala Najmeddine, K EI Khamlichi Drissi, Christophe Pasquier, Claire Faure, Kamal Kerroum, Alioune Diop, Thierry Jouannet, and Michel Michou, "State of art on load monitoring methods," in Power and Energy Conference, 2008. PECon 2008. IEEE 2nd International.IEEE, 2008, pp. 1256-1258

[11] Yi Du, Liang Du, Bin Lu, Ronald Harley, and Thomas Habetler, "A review of identification and monitoring methods for electric loads in commercial and residential buildings," in Energy Conversion Congress and Exposition (ECCE), 2010 IEEE. IEEE, 2010, pp. 4527-4533.

[12] Michael Zeifman and Kurt Roth, "Nonintrusive appliance load monitoring: Review and outlook," Consumer Electronics, IEEE Transactions on, vol. 57, no. 1, pp. 76-84,2011.

[13] R. Bonfigli, S. Squartini, M. Fagiani, and F. Piazza, "Unsupervised algorithms for non-intrusive load monitoring: An up-to-date overview," in Environment and Electrical Engineering (EEEIC), 2015 IEEE 15th International Conference on, June 2015, pp. 1175-1180

[14] J. Z. Kolter and M. J. Johnson, "Redd: A public data set for energy disaggregation research," in Workshop on Data Mining Applications in Sustainability (SIGKDD), San Diego, CA, vol. 25. Citeseer, 2011, pp.59-62.

[15] K. Anderson, A. Ocneanu, D. Benitez, D. Carlson, A.Rowe, and M. Berges, "Blued: A fully labeled public dataset for event-based nonintrusive load monitoring research," in Proceedings of the 2nd KDD workshop on data mining applications in sustainability (SustKDD), 2012, pp. 1-5.

[16] J. Gao, S. Giri, E. C. Kara, and M. Berg'es, "Plaid: a public dataset of high-resoultion electrical appliance measurements for load 
identification research: demo abstract," in Proceedings of the 1st ACM Conference on Embedded Systems for Energy-Efficient Buildings. ACM, 2014, pp.198-199.

[17] Kahl, Matthias \& Haq, Anwar \& Kriechbaumer, Thomas \& Jacobsen, Hans-Arno. (2016). WHITED - A Worldwide Household and Industry Transient Energy Data Set.

[18] Picon, Thomas \& Nait-Meziane, Mohamed \& Ravier, Philippe \& Lamarque, Guy \& Novello, Clarisse \& Le Bunetel, Jean-Charles \& Raingeaud, Yves. (2016). COOLL: Controlled On/Off Loads Library, a Public Dataset of High-Sampled Electrical Signals for Appliance Identification.

[19] Nait-Meziane, Mohamed \& Picon, Thomas \& Ravier, Philippe \& Lamarque, Guy \& Le Bunetel, Jean-Charles \& Raingeaud, Yves. (2016). A measurement system for creating datasets of on/offcontrolled electrical loads. 1-5. 10.1109/EEEIC.2016.7555847

[20] Nait-Meziane, Mohamed \& Ravier, Philippe \& Lamarque, Guy \& Le Bunetel, Jean-Charles \& Raingeaud, Yves. (2017). High accuracy event detection for Non-Intrusive Load Monitoring. 10.1109/ICASSP.2017.7952597. 